\title{
Can disc diffusion susceptibility tests assess the antimicrobial activity of engineered nanoparticles?
}

\author{
Angeliki Kourmouli • Marco Valenti • \\ Erwin van Rijn • Hubertus J. E. Beaumont • \\ Olga-Ioanna Kalantzi • Andreas Schmidt-Ott • \\ George Biskos $\mathbb{D}$
}

Received: 27 September 2017 / Accepted: 5 February 2018 /Published online: 2 March 2018

(C) The Author(s) 2018. This article is an open access publication

\begin{abstract}
The use of disc diffusion susceptibility tests to determine the antibacterial activity of engineered nanoparticles (ENPs) is questionable because their low diffusivity practically prevents them from penetrating through the culture media. In this study, we investigate the ability of such a test, namely the Kirby-Bauer disc diffusion test, to determine the antimicrobial activity of $\mathrm{Au}$ and Ag ENPs having diameters from 10 to $40 \mathrm{~nm}$ on Escherichia coli
\end{abstract}

\section{A. Kourmouli $\cdot$ M. Valenti $\cdot$ A. Schmidt-Ott Faculty of Applied Sciences, Delft University of Technology, 2628-BL Delft, The Netherlands}

\section{A. Kourmouli · O.-I. Kalantzi \\ Department of Environment, University of the Aegean, 81100 Mytilene, Greece \\ E. van Rijn · H. J. E. Beaumont}

Kavli Institute of Nanoscience, Department of Bionanoscience,

Delft University of Technology, 2628-CJ Delft, The Netherlands

G. Biskos $(\square)$

Faculty of Civil Engineering and Geosciences, Delft University of Technology, 2628-CN Delft, The Netherlands

e-mail: g.biskos@tudelft.nl

e-mail: g.biskos@cyi.ac.cy

\section{G. Biskos}

Energy, Environment and Water Research Center, The Cyprus Institute, 2121 Nicosia, Cyprus

Present Address:

A. Kourmouli

The Birmingham Institute of Forest Research (BIFoR),

School of Geography Earth and Environmental Sciences,

University of Birmingham, Birmingham B15 2TT, UK cultures. As anticipated, the tests did not show any antibacterial effects of Au nanoparticles (NPs) as a result of their negligible diffusivity through the culture media. Ag NPs on the other hand exhibited a strong antimicrobial activity that was independent of their size. Considering that $\mathrm{Ag}$, in contrast to $\mathrm{Au}$, dissolves upon oxidation and dilution in aqueous solutions, the apparent antibacterial behavior of Ag NPs is attributed to the ions they release. The KirbyBauer method, and other similar tests, can therefore be employed to probe the antimicrobial activity of ENPs related to their ability to release ions rather than to their unique size-dependent properties.

Keywords Engineered nanoparticles · Disc diffusion method · Silver nanoparticles · Gold nanoparticles . Antimicrobial activity Aerosol-based nanoparticle synthesis

\section{Introduction}

Engineered nanoparticles (ENPs) have numerous applications in medicine including treatments for fungal and HIV infections, as well as various types of cancer (Zhang et al. 2008). Compared to their large-particle counterparts, ENPs also exhibit enhanced antimicrobial activity and are therefore employed in many hygiene products (Choi et al. 2008; Sotiriou and Pratsinis 2010). A number of methods, including the Kirby-Bauer disc diffusion susceptibility technique, have been used to determine the antibacterial activity of ENPs (Oberdörster et al. 2005). The challenge of assessing the toxicity of ENPs, 
however, lies in the difficulty to discriminate between size-related effects caused by direct particle-cell interactions, from those caused by the potential compounds they release upon dissolution to the culture media.

An increasing number of studies have employed the Kirby-Bauer method for assessing the antibacterial activity of ENPs. Guzman et al. (2012) as well as Devi and Bhimba (2012) used this technique to show that Ag nanoparticles (NPs) smaller than $30 \mathrm{~nm}$ produced by standard liquid-based methods can have strong antibacterial effects. Geethalakshmi and Sarada (2012) used the same method to show that $\mathrm{Ag}$ and $\mathrm{Au}$ NPs larger than $30 \mathrm{~nm}$ produced by extracts from Trianthema decandra roots also have enhanced antimicrobial activity. More recently, Bhuyan et al. (2017) used the Kirby-Bauer technique for assessing the antimicrobial activity of Ag and Au NPs produced by extracts from Paederia foetida Linn. Their results showed that Au NPs did not exhibit any antimicrobial activity against all pathogens, contrary to their Ag counterparts. It should be noted here that these studies do not distinguish between the toxic behavior caused by direct nanoparticlecell interactions and those induced by potential dissolution of toxic species from the surface of the NPs. This point is particularly important for the interpretation of the results since, as will be demonstrated below, only species released from the NPs can penetrate into the culture media and inhibit the growth of the cells.

The Kirby-Bauer method relies on the diffusion of the test substance (i.e., the ENPs in the aforementioned studies) from the filter discs to the bacterial cultures (cf. Methods section for more details). The diffusivity of NPs having diameters larger than $10 \mathrm{~nm}$ in culture media used in diffusion susceptibility tests is in the order of $10^{-11} \mathrm{~m}^{2} / \mathrm{s}$. This is at least one order of magnitude lower compared to the respective diffusivity of common antibiotics for which such tests are commonly used. As a consequence, ENPs do not travel far from the deposition discs to physically interact with the bacterial cells, raising doubts whether the method can probe antibacterial activity related to their size. To the best of our knowledge, this has not been considered by other studies reported in the literature thus far.

The aim of this study is to test the hypothesis that the Kirby-Bauer method only detects antibacterial effects of ENP-derived dissolved compounds. To this end, we examined the antibacterial activity of pure ENPs composed of $\mathrm{Au}$ and $\mathrm{Ag}$ (i.e., two metals that behave differently in aqueous media but have a toxic behavior at the nanoscale; cf. Sadeghi et al. 2010; Peretyazhko et al. 2014; Ilaria et al. 2015; Shrivastava et al. 2016;
Chandran et al. 2017) on Escherichia coli cultures. ENPs had diameters from 10 to $40 \mathrm{~nm}$, and to make the results comparable among all tests, we kept their total surface concentration constant in all our samples.

\section{Methods}

Particle production Pure (ligand-free) Au and Ag NPs were synthesized by vapor nucleation in $\mathrm{N}_{2}$ gas (99.999\% purity) using a spark-discharge particle generator (cf. Fig. 1). This method, described in detail by Tabrizi et al. (2009) and more recently by Pfeiffer et al. (2014), can be used to synthesize well-defined NPs with good control over their composition, including both single-component or mixed/alloy NPs of high purity (Feng et al. 2018). What is also important for employing this technique to produce samples for toxicity tests is that combined with a Differential Mobility Analyzer (DMA; i.e., a classifier that selects particles based on their electrical mobility; Knutson and Whitby 1975), it can produce uniformly-sized NPs having diameters within a very narrow range (i.e., nearly monodisperse NPs) as has been illustrated by a number of recent studies (Feng et al. 2015; Feng et al. 2016; Valenti et al. 2017).

In brief, two opposing cylindrical Ag or Au electrodes (MaTecK GmbH, Germany; 99.99\% purity) are placed a few millimeters apart. Repeated electrical breakdowns form when a high potential difference is applied between the two electrodes, resulting in a nearly-continuous evaporation of material from their surface. A high-purity $\mathrm{N}_{2}$ gas flow passed between the two electrodes dilutes and cools the vapor cloud to form pure $\mathrm{Ag}$ or Au NPs of different sizes and morphologies (i.e., from spherical to highly agglomerated particles) depending on the flow rate and electrical energy used to form the sparks (Feng et al. 2015). A tube oven maintained at $\sim 1000{ }^{\circ} \mathrm{C}$ was used downstream the sparkdischarge generator to sinter the agglomerates to spherical particles. An example Transmission Electron Microscopy (TEM) image of 15-nm Ag NPs produced by the setup used in this study is provided in Fig. 2, verifying the capability of the system to produce nearly monodisperse spherical particles. Similar results were obtained for NPs having sizes within the entire range invested here, including for the Au NPs. In addition to that, X-ray photoelectron spectroscopy confirmed that the resulting NPs consisted purely of Au or Ag depending on the electrodes used in the spark-discharge 


\section{Spark \\ Discharge}
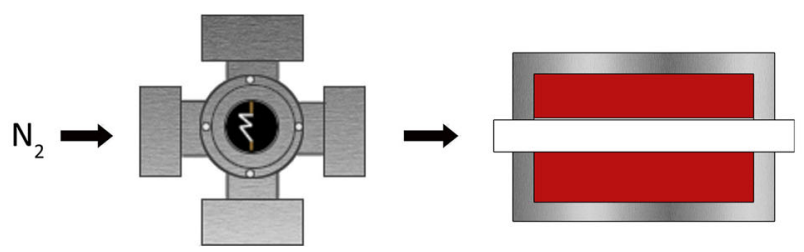

Fig. 1 Schematic layout of the apparatus used for the production of ENPs. High-purity Au or Ag agglomerates were produced by spark ablation and sintered to spherical particles in a tube oven. Monodisperse fractions of the resulted spherical particles were

generator (data not shown here). Compared to classical wet-chemistry methods for synthesizing ENPs, spark ablation in the gas phase delivers NPs of higher purity (Biskos et al. 2008), which is crucial for studying their interactions with living cells.

To select particles of a specific size, we used a custom-made DMA having an effective length of $114 \mathrm{~mm}$ and diameters of the outer and inner electrodes of 19.54 and $9.35 \mathrm{~mm}$, respectively (cf. Barmpounis et al. 2016, and the supplementary information therein, for additional details). After size selection, the particles were collected on glass-fiber filter discs with a diameter of $47 \mathrm{~mm}$. A Condensation Particle Counter (CPC; TSI

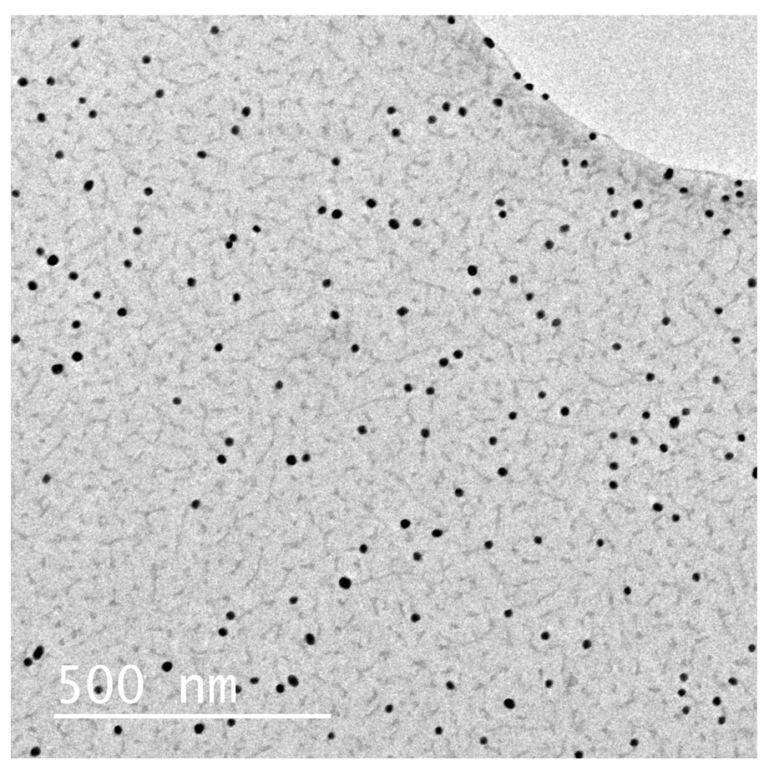

Fig. 2 Transmission electron microscope image of the nearlymonodisperse Ag NPs produced by the experimental setup used in this study
DMA $\begin{array}{cc}\text { Filter } \\ \text { Sampler }\end{array}$

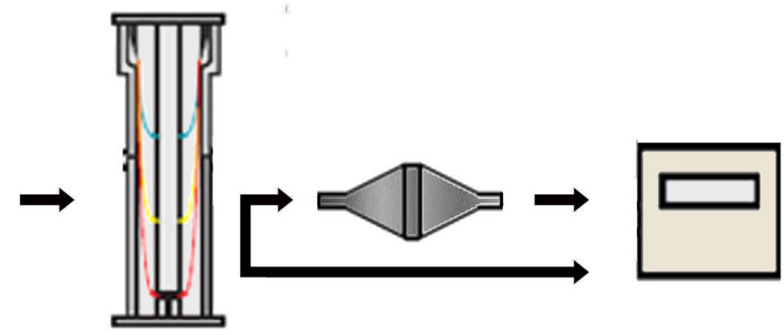

selected by a DMA and deposited on glass fiber filters. The concentration of the monodisperse particles downstream the DMA and the filter sampler was continuously monitored by a CPC

Model 3025; Agarwal and Sem 1980) was used to measure the aerosol nanoparticle concentration in order to control the total surface area of the collected particles, which was kept constant for all the filters prepared (i.e., $\sim 14.5 \times 10^{-5} \mathrm{~m}^{2}$ for both $\mathrm{Ag}$ and Au ENPs).

Toxicity experiments Cultures of $E$. coli were cultivated on Mueller-Hinton (MH2) agar plates as illustrated in Fig. 3. Bacteria samples from one or two over-night grown colonies were suspended in a test tube containing nutrient broth (i.e., lysogeny broth). The turbidity (expressed as optical density; OD) of the bacterial suspensions were measured with an optical spectrophotometer $(\lambda=600 \mathrm{~nm})$ and adjusted to 0.25 or 0.50 . A sterilized cotton swab was immersed in the resulting suspension, and a lawn of bacteria was applied on the agar plates.

Nanoparticle-laden filter discs with a diameter of $5 \mathrm{~mm}$ were cut out of the 47-mm filters and applied on top of the bacterial cultures. The side containing the particles was facing downwards to ensure direct interaction with the agar and the bacteria, and the samples were incubated at $37^{\circ} \mathrm{C}$ for $48 \mathrm{~h}$. The inhibition zones around the filters were measured every $12 \mathrm{~h}$ according to the Kirby-Bauer test protocol (Bauer et al. 1966).

\section{Results and discussion}

Table 1 shows the size of the inhibition zones around the filters loaded with $\mathrm{Ag}$ and Au ENPs having diameters of 10,20 , and $40 \mathrm{~nm}$. Figure 4 shows images of the E. coli cultures with the deposited ENP-laden filter discs after 48 h. The cultures exposed to Au NPs did not exhibit 
Fig. 3 Schematic layout of the experimental procedure used for the toxicity experiments. Fivemillimeter nanoparticle-laden filter discs were cut out of the original filter (step 1) while $E$. coli cultures were laid on the $\mathrm{MH} 2$ agar plates (step 2). The filter discs were then deposited on the E. coli cultures with the nanoparticle deposition side facing down (step 3). The size of the inhibition zones around the filter discs was measured after $48 \mathrm{~h}$ of incubation (step 4)

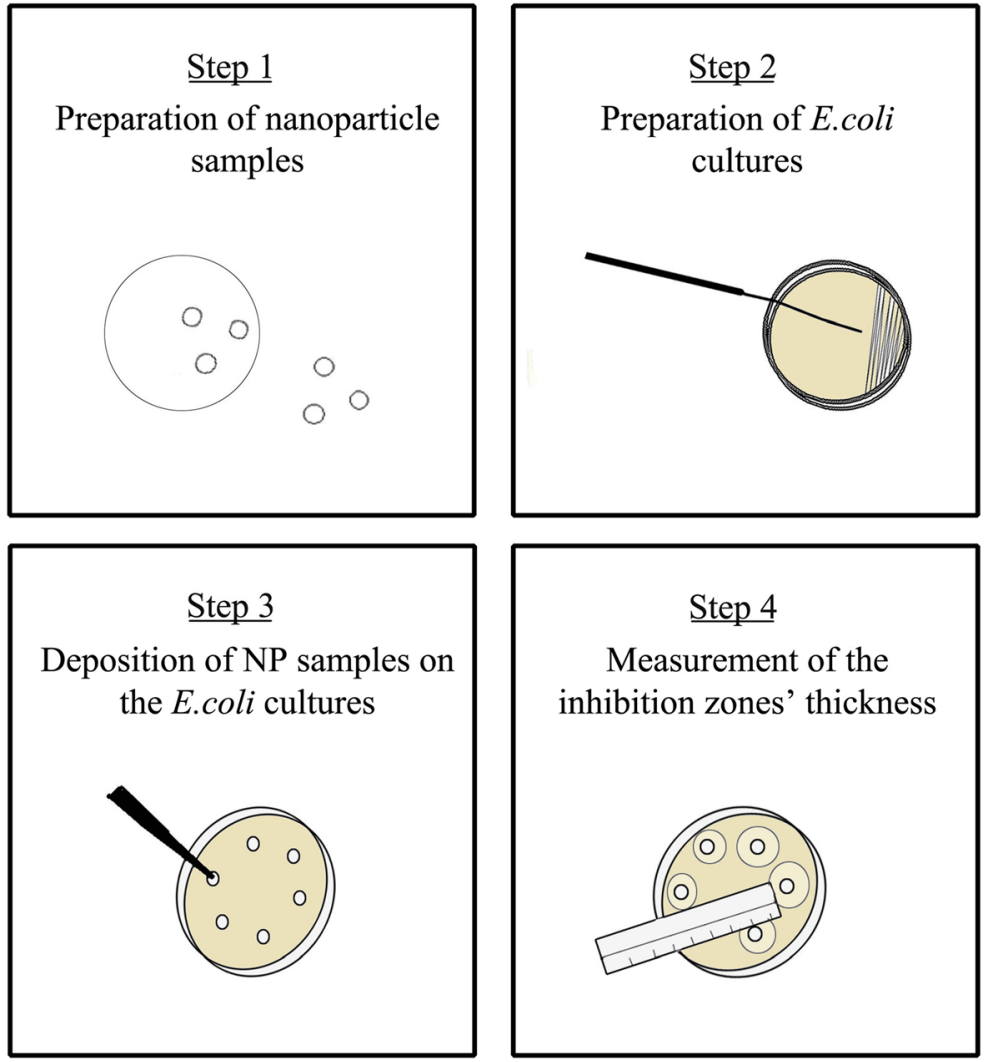

any inhibition zones around the filters, indicating that they do not have any antibacterial properties. The tests with the Ag NPs, on the other hand, exhibited inhibition zones of the same size for all particle diameters tested. It should be noted here again that the total surface of the particles on the filter samples was kept the same in order to ensure that the potential release rates of the ions (or of any other substance from the surface of the NPs) was the same throughout all tests (cf. Liu and Hurt 2010; Lee et al. 2012).

Although the results summarized in Table 1 can be interpreted as a difference in the antibacterial

Table 1 Size of inhibition zones ( $\mathrm{mm}$ ) measured from the edge of the filter discs to the edge of the bacterial lawn, around the nanoparticle-laden filters

\begin{tabular}{llllll}
\hline Particle & \multicolumn{2}{l}{ Ag Nanoparticles } & & \multicolumn{2}{l}{ Au Nanoparticles } \\
\cline { 2 - 3 } \cline { 5 - 6 } Diameter (nm) & (OD: 0.5) & (OD: 0.25) & & (OD: 0.5) & (OD: 0.25) \\
\hline 10 & 2 & 1 & 0 & 0 \\
20 & 2 & 1 & 0 & 0 \\
40 & 2 & 1 & 0 & 0 \\
\hline
\end{tabular}

effects between Ag and Au ENPs on E. coli, they should be treated with caution. The most plausible explanation of our observations is related to the release of ions from the ENPs. In contrast to $\mathrm{Au}$, $\mathrm{Ag}$ releases ions upon oxidation and suspension in aqueous solutions (e.g., Wijnhoven et al. 2009; Bae et al. 2010; Le Ouay and Stellacci 2015). Considering that $\mathrm{Ag}^{+}$ions have a strong antimicrobial activity (Zhao and Stevens 1998; Feng et al. 2000; Xiu et al. 2012) that can inhibit bacterial growth, the observed discrepancies between the measurements using Ag and Au NPs can be attributed to the difference of this intrinsic property for the two materials.

The hypothesis that the $\mathrm{Ag}^{+}$ions are the antimicrobial agents in these tests is also consistent with the fact that the size of the inhibition zones around the Agnanoparticle-laden filters was the same for all particle sizes tested. This is because the total nanoparticle surface concentration was kept constant throughout all samples, ensuring that the ion release rate was the same in all the tests as discussed above. Additional supporting evidence that the $\mathrm{Ag}^{+}$ions are responsible for inhibition 

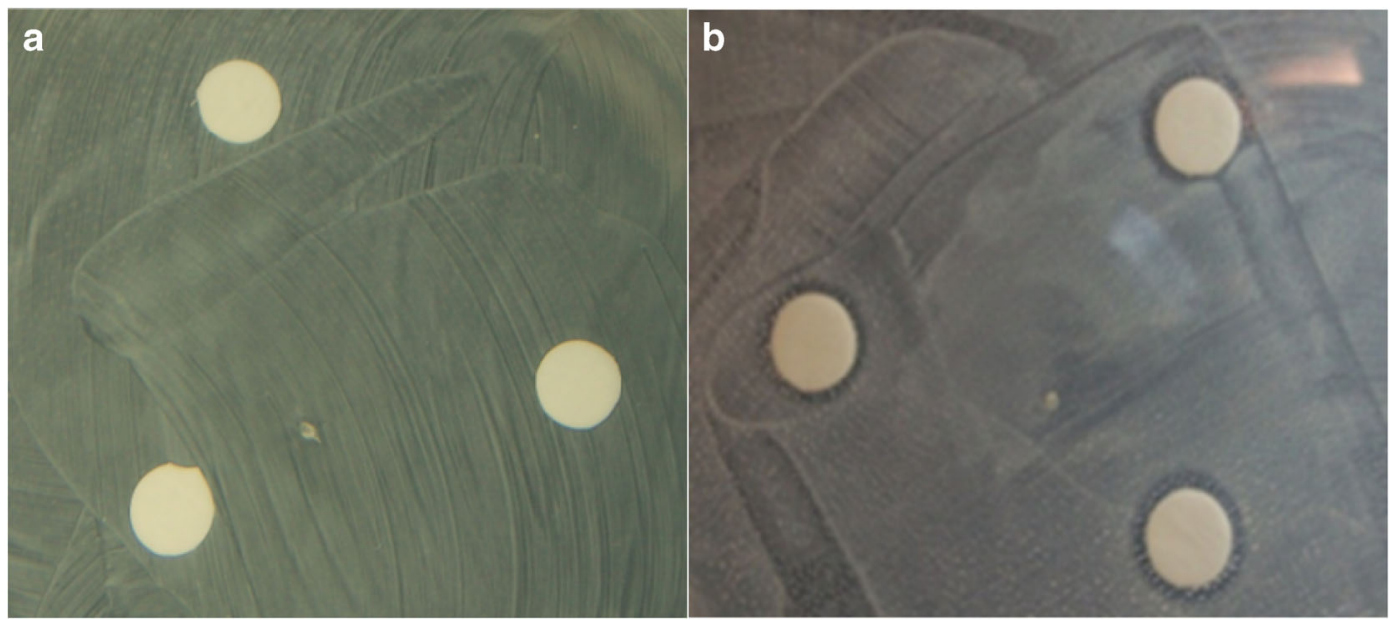

Fig. 4 Images of grown E. coli cultures with ENP-laden filter discs containing 10-nm Au (a) and Ag (b) ENPs. In both cases, the surface concentration of the particle depositions was the same (i.e., $\sim 14.5 \times 10^{-5} \mathrm{~m}^{2}$ )

of bacterial growth in these tests is the actual size of the inhibition zones around the Ag-nanoparticle filters. Considering that those were up to a couple of millimeters wide, this observation would require a toxic species having a diffusivity in the order of $10^{-9} \mathrm{~m}^{2} / \mathrm{s}$ in the solution of the culture. This value is of the same order as the diffusivity of silver ions in the culture medium, and definately much higher than that of ENPs larger than $10 \mathrm{~nm}$ that are used in our experiments.

In conclusion, our observations show that the Kirby-Bauer method (and in principle other similar disc diffusion susceptibility tests) can be used for assessing the antimicrobial activity of ENPs. It should be noted, however, that the probed antibacterial activity is related to the potential of the NPs to release toxic and highly diffusive ions (or other soluble species) into the culture growth medium, which in the case of our experiments was due to the dissolution of $\mathrm{Ag}$, rather than the physical interaction with the nanometer-sized particles. This characteristic can explain the difference in the antibacterial behavior of $\mathrm{Au}$ and $\mathrm{Ag}$ ENPs tested in this study, and can be used in a systematic way for distinguishing between ion and nanosize toxicity of ENPs.

Acknowledgements AK acknowledges the Erasmus Placement Program for funding her visit at TU Delft.

\section{Compliance with ethical standards}

Conflict of interest The authors declare that they have no conflict of interest.
Open Access This article is distributed under the terms of the Creative Commons Attribution 4.0 International License (http:// creativecommons.org/licenses/by/4.0/), which permits unrestricted use, distribution, and reproduction in any medium, provided you give appropriate credit to the original author(s) and the source, provide a link to the Creative Commons license, and indicate if changes were made.

\section{References}

Agarwal JK, Sem GJ (1980) Continuous flow, single-particlecounting condensation nucleus counting. J Aerosol Sci 11: 343-357

Barmpounis K, Maisser A, Schmidt-Ott A, Biskos G (2016) Lightweight differential mobility analyzers: towards new and inexpensive manufacturing methods. Aerosol Sci Technol 50:ii-V

Bae E, Park HJ, Lee J, Kim Y, Yoon J, Park K, Choi K, Yi J (2010) Bacterial cytotoxicity of the silver nanoparticle related to physicochemical metrics and agglomeration properties. Environ Toxicol Chem 29:2154-2160

Bauer AW, Kirby WM, Sherris JC, Turck M (1966) Antibiotic susceptibility testing by a standardized single disk method. Am J Clin Pathol 45:493-496

Bhuyan B, Paul A, Paul B, Dhar SS, Dutta P (2017) Paederia foetida Linn. promoted biogenic gold and silver nanoparticles: synthesis, characterization, photocatalytic and in vitro efficacy against clinically isolated pathogens. J Photochem Photobiol B Biol 173:210-215

Biskos G, Vons VA, Yurteri CU, Schmidt-Ott A (2008) Generation and sizing of particles for aerosol-based nanotechnology. KONA Powder and Particle 26:13-35

Chandran P, Riviere JE, Monteiro-Riviere NA (2017) Surface chemistry of gold nanoparticles determines the biocorona composition impacting cellular uptake, toxicity and gene 
expression profiles in human endothelial cells. Nanotoxicology 11:507-519

Choi O, Deng KK, Kim NJ, LJr R, Surampalli RY, Hu Z (2008) The inhibitory effects of silver nanoparticles, silver ions and silver chloride colloids on microbial growth. Water Res 42: 3066-3074

Devi JS, Bhimba BV (2012) Silver nanoparticles: antibacterial activity against wound isolates \& in vitro cytotoxic activity on human Caucasian colon adenocarcinoma. Asian Pacific Journal of Tropical Diseases 2:87-93

Feng QL, Wu J, Chen GQ, Cui FZ, Kim TN, Kim JO (2000) A mechanistic study of the antibacterial effect of silver ions on Escherichia coli and Staphylococcus aureus. J Biomed Mater Res 52:662-668

Feng F, Ramlawi N, Biskos G, Schmidt-Ott A (2018) Internally mixed nanoparticles from oscillatory spark ablation. Aerosol Science and Technology in press

Feng J, Huang L, Ludvigsson L, Messing ME, Maisser A, Biskos G, Schmidt-Ott A (2016) General approach to the evolution of singlet nanoparticles from a rapidly quenched point source. J Phys Chem C 120:621-630

Feng J, Biskos G, Schmidt-Ott A (2015) Toward industrial scale synthesis of ultrapure singlet nanoparticles with controllable sizes in a continuous gas-phase process. Sci Rep 5:15788

Ilaria F, Iole V, Cesare C, Maria Vittoria R (2015) How toxic are gold nanoparticles? The state-of-the-art. Nano Res 8(6): 1771-1799

Geethalakshmi R, Sarada DV (2012) Gold and silver nanoparticles from Trianthema decandra: synthesis, characterization, and antimicrobial properties. Int J Nanomedicine 7:5375-5384

Guzman M, Dille J, Godset S (2012) Synthesis and antibacterial activity of silver nanoparticles against gram-positive and gram-negative bacteria. Nanomedicine 8:37-45

Knutson EO, Whitby KT (1975) Aerosol classification by electric mobility: apparatus, theory and applications. J Aerosol Sci 6: 443-451

Le Ouay B, Stellacci F (2015) Antibacterial activity of silver nanoparticles: a surface science insight. NanoToday 10: 339-354

Lee YJ, Kim J, Oh J, Bae S, Lee S, Hong IS, Kim SH (2012) Ionrelease kinetics and ecotoxicity effects of silver nanoparticles. Environ Toxicol Chem 31(1):155-159
Liu J, Hurt RH (2010) Ion release kinetics and particle persistence in aqueous Nano-silver colloids. Environ Sci Technol 44: 2169-2175

Oberdörster G, Oberdörster E, Oberdörster J (2005) Nanotoxicology: an emerging discipline evolving from studies of ultrafine particles. Environ Health Perspect 113:823-840

Peretyazhko TS, Zhang Q, Colvin VL (2014) Size-controlled dissolution of silver nanoparticles at neutral and acidic $\mathrm{pH}$ conditions: kinetics and size changes. Environ Sci Technol 48(20): 11954-11961

Pfeiffer TV, Feng J, Schmidt-Ott A (2014) New developments in spark production of nanoparticles. Adv Powder Technol 25: 56-70

Shrivastava R, Kushwaha P, Bhutia YC, Flora SJS (2016) Oxidative stress following exposure to silver and gold nanoparticles in mice. Toxicol Ind Health 32(8):1391-1404

Sadeghi B, Jamali M, Kia S, Amini nia A, Ghafari S (2010) Synthesis and characterization of silver nanoparticles for antibacterial activity. International Journal of Nano Dimension 1(2):119-124

Sotiriou GA, Pratsinis SE (2010) Antibacterial activity of nanosilver ions and particles. Environ Sci Technol 44: 5649-5654

Tabrizi NS, Ullmann M, Vons VA, Lafont U, Schmidt-Ott A (2009) Generation of nanoparticles by spark discharge. J Nanopart Res 11:315-332

Valenti M, Venugopal A, Tordera D, Jonsson PM, Biskos G, Schmidt-Ott A, Smith WA (2017) Hot carrier generation and extraction of plasmonic alloy nanoparticles. ACS Photonics 4:1146-1152

Wijnhoven SWP, Peijneburg WJGM, Herberts CA, Hagens WI, Oomen AG, Heugens EHW et al (2009) Nano-silver-a review of available data and knowledge gaps in human and environmental risk assessment. Nanotoxicology 3:1-30

Xiu ZM, Zhang QB, Puppala HL, Colvin VL, Alvarez PJ (2012) Negligible particle-specific antibacterial activity of silver nanoparticles. Nano Lett 12(8):4271-4275

Zhang L, Gu FX, Chan JM, Wang AZ, Langer RS, Farokhzad OC (2008) Nanoparticles in medicine: therapeutic applications and developments. Clin Pharmacol Ther 83:761-769

Zhao G, Stevens E (1998) Multiple parameters for the comprehensive evaluation of the susceptibility of Escherichia coli to the silver ion. Biometals 11:27-32 